\title{
Internet of Things in Sleep Monitoring: An Application for Posture Recognition Using Supervised Learning
}

\author{
Georges Matar ${ }^{1}$, Jean-Marc Lina ${ }^{1,2}$, Julie Carrier ${ }^{2}$, Anna Riley ${ }^{3}$, Georges Kaddoum ${ }^{1}$ \\ ${ }^{1}$ Biomedical Information Processing Laboratory LATIS, École De Téchnologie Supérieure, Montreal (Quebec), Canada \\ ${ }^{2}$ Center For Advanced Research In Sleep Medecine, Montreal (Quebec), Canada \\ ${ }^{3}$ Riley's 321Sleep, Tecumseh, Ontario, Canada \\ \{georges.matar.1@ens.etsmtl.ca, jean-marc.lina@etsmtl.ca, georges.kaddoum@etsmtl.ca\}
}

\begin{abstract}
In this paper, we propose an Internet of Things (IoT) system application for remote medical monitoring. The body pressure distribution is acquired through a pressure sensing mattress under the person's body, data is sent to a computer workstation for processing, and results are communicated for monitoring and diagnosis. The area of application of such system is large in the medical domain making the system convenient for clinical use such as in sleep studies, non or partial anesthetic surgical procedures, medical-imaging techniques, and other areas involving the determination of the body-posture on a mattress. In this vein, a novel method for human body posture recognition that consists in providing an optimal combination of signal acquisition, processing, and data storage to perform the recognition task in a quasi-real-time basis. A supervised learning approach was used to build a model using a robust synthetic data. The data has been generated beforehand, in a way to enhance and generalize the recognition capability while maintaining both geometrical and spatial performance. Low-cost and fast computation per sample processing along with autonomy, make the system suitable for long-term operation and IoT applications. The recognition results with a Cohen's Kappa coefficient $\kappa=0.866$ was satisfactorily encouraging for further investigation in this field.
\end{abstract}

\section{INTRODUCTION}

The continuous advances in medical technologies in the recent decades, and the efficiency improvements on health care services provided with the emerging telemedicine, have made the patient monitoring a crucial concern. The vast range of patient monitoring systems includes tracking of vital signs, physiological parameters, or physical behavior of the patient. A particular type of physical monitoring is the detection of the body posture for a person sleeping on a mattress. Such monitoring could be useful for sleep studies, non or semianesthetic surgical procedures, and after surgical monitoring e.g., orthopedic surgery where the success of the surgery is conditioned on the patient that should maintain specific postures for days or weeks after the surgical procedure, medical imaging techniques, and other areas involving the determination of the body-posture on a mattress [1], [2]. Conventional means of physical monitoring are mostly based under the surveillance of professionals themselves which requires considerable cost and resources. Alternatives are conceived such as telesurveillance, based on the monitoring of the patient using camera recording, which also suffer from some limitations such as short term applications, e.g., imaging techniques or surgical procedures, a spatial field around the patient should remain isolated and not attained by professionals and/or materials, to maintain the field of view required for camera recording.

Such constraints prompt the need for a new system to monitor the posture of the patient in a different mean, where the constraints on both the person being monitored and the professional are as few as possible.

IoT (Internet of Things) is a generic term to denote objects connected to each other for several uses in a wide range of fields to measure and exchange data from real world i.e. medical field, and home automation [3]. A typical architecture of an IoT application can be defined by three main components: application layer in which the data processing and service providing to the end user are performed, transmission layer where long distance communications are aimed, and the perception layer where the acquisition, processing and local communication through Wireless Sensor Networks (WSNs) of the physical (real) data are involved [4]. The use of IoT communication has been increasing and developing to cover more applications in the medical field including patient monitoring [5], [6]. The recent IoT research directions in the medical field can be divided in four main categories: network architectures and platforms, interoperability, new services and applications, and security [7].

The use of body pressure mapping has been involved in few applications such as advising clients to choose the mattress that has the best corresponding elasticity and texture/materials properties to support his body, and clearly this is evaluated based on the equally distributed pressure of the human body on the mattress [8], [9], and monitoring the body pressure distribution during sleep for hospitalized patients to prevent bed sores and ulcers [10]. Hsu et al [11] proposed a complete solution to provide automatic body support for patients to prevent the occurrence of ulcers caused by long-time same sleeping position. Pressure sensor mattresses used to measure the pressure distribution of a human body existing in the market are composed of a matrix-form of pressure sensors 
implemented on the surface of a mattress. A Bluetooth communication module is embedded in the system to ensure data communication with a computer acting as a workstation used for analysis, storage, and communication through web to medical centers and professionals. Different types of pressure sensors are used in pressure sensing mattresses. The most widely used type in such application is the Force Sensing Resistor (FSR) [8].

In this paper, we discuss the feasibility of integrating IoT in a medical application by evaluating both technical and clinical constraints, and assessing core IoT requirements. Moreover, the posture of the person sleeping on a mattress is of interest, we propose a method using a supervised learning SVM (Support Vector Machine) algorithm to deal with posture recognition as a binary pattern recognition problem using a minimal information i.e. binary images of the patient on the mattress. Accordingly, retaining a fast and efficient processing with a low computation cost to meet the requirements of IoT communication. An IoT communication application and scenario is proposed to make sure that the operation is done remotely for ultimate unobtrusivity for both person being monitored, and monitoring parties.

The materials and methods used in the implementation are discussed in section II, a discussion of the obtained results in section III, and a brief conclusion along with perspectives and future work are presented in section IV.

\section{MATERials AND METHODS}

In this section, the steps behind the algorithm proposed are presented with the scientific rationale behind. We start with a quick terminology to clarify some terms for the convenience of the reader, then the methods used to deal with database, preprocessing, implementation, validation, and discrimination of the fourth posture are all discussed in details.

\section{A. Database}

As mentioned in section I, different types of pressure sensors are used in body pressure distribution. In this paper, we used as a reference, the mattress Tactilus [12], made by Sensor Products Inc as starting point. The system architecture is shown in Figure 1. Reasons for choosing this mattress are the following:

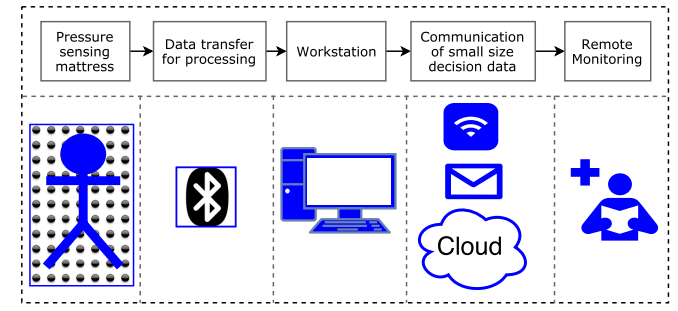

Fig. 1: System architecture

- Sampling frequency up to $26 \mathrm{~Hz}$, which permits monitoring of short ultradian periodic changes inside the body, reflected as pressure distribution changes on the mattress, such as the respiration.
- Several output file are permitted. In our case, .png images containing the raw data were considered. Also video scenes of pressure variation over time can be recorded, making possible to extend the application to cover the aforementioned periodic pressure distribution changes.

- Wireless communication making the data sent to a workstation wirelessly [12]. A possibility to operate on battery for a duration period predetermined by the user (even more than whole sleeping nights), making possible the system autonomy in terms of power and data.

For the sake of efficiency, the classification is done using the less possible pressure information, i.e. only reduced dimensions binary images are used instead of the full pressure information images, with an algorithm involving the less computation possible for the following consideration: computation will be done in a quasi real-time basis. We are interested of decreasing the computation cost, and computation time per iteration, as the computation will be a repetitive, iterative, and continuous but at the same time, must be fast and efficient to respond to many clinical and IoT applications requirements. Based on the previous requirements, the pressure mapping images used are binary i.e., permitted individual sensor output pressure values are 0 or 1 . Samples from the mattress are used to generate training data. It is straightforward that for prone and supine positions, a simple discrimination cannot be done using binary images, because the shape of the body on the mattress for both positions will be very close. That is why prone and supine position are considered as a one position, named $\mathrm{s} / \mathrm{p}$ position. Hence, when building the training data, three positions were considered: s/p, left lateral, and right lateral. For each of the three positions, a set containing 74 samples of $14 \times 32$ pixels binary images was used, to form a total of 222 images for training. As shown in equation (1), for each image $\boldsymbol{\Phi}^{(\mathbf{i})}, i=1,2 \ldots, N=222$, the $\mathbf{m} \times \mathbf{n}$ matrix of pressure values was converted to a feature vector $\Phi_{11}^{(i)} \ldots \Phi_{m n}^{(i)}$ having a 448 dimensions where $\mathrm{m}=14$ and $\mathrm{n}=32$, let $M=m \times n$, hence the size of the data matrix $\boldsymbol{\Phi}$ is $N \times M$. The group of training images form the database matrix $\boldsymbol{\Phi}$.

$$
\boldsymbol{\Phi}=\left[\begin{array}{ccccc}
\Phi_{11}^{(1)} & \Phi_{12}^{(1)} & \Phi_{13}^{(1)} & \ldots & \Phi_{m n}^{(1)} \\
\Phi_{11}^{(2)} & \Phi_{12}^{(2)} & \Phi_{13}^{(2)} & \ldots & \Phi_{m n}^{(2)} \\
\ldots \ldots \ldots \ldots \ldots \ldots \ldots \ldots \ldots \ldots \ldots . \\
\Phi_{11}^{(N)} & \Phi_{12}^{(N)} & \Phi_{13}^{(N)} & \ldots & \Phi_{m n}^{(N)}
\end{array}\right]
$$

The test database consists of 90 samples of different positions body pressure distribution images, totally different of the training samples. The orientation and location of both body and limbs, hence the pose were ignored considering that as long as the patient remains sleeping on the mattress, the interest is to detect the position. Graphical display of output pressure image can illustrate the body location and pose but they are out of interest.

\section{B. Preprocessing and Features Extraction}

In order to make use of the binary pressure distribution images in building the model, they were converted to variables 
and saved in the workspace. A dimensionality reduction procedure was conducted to keep only the useful and discriminative information of the data.

1) Principal Component Analysis (PCA): PCA is one of the most widespread methods in data analysis. It consists of applying orthogonal transformations to variables that are assumed to be possibly correlated, which are our observations, in order to make them linearly uncorrelated, aiming to preserve the most variations between the data variables and avoid redundancy. Hence the first principal component is the one that accounts for the most data variability, while the second accounts for less variance, and is orthogonal to the previous one. The resulting uncorrelated variables form then an orthogonal basis set.

The mean of each column of the matrix $\boldsymbol{\Phi}$ is calculated, then subtracted from each one of the feature values, in order to center the data, let the centered database matrix be $\overline{\mathbf{\Phi}}$. Let $\mathbf{Y}=$ $\frac{1}{\sqrt{N-1}} \overline{\boldsymbol{\Phi}}^{\mathbf{T}}$, and $\mathbf{S}_{\overline{\mathbf{\Phi}}}=\mathbf{Y}^{\mathbf{T}} \mathbf{Y}$ is the covariance matrix of $\boldsymbol{\Phi}$, where $\mathbf{T}$ is the transpose operator. The principal components of $\overline{\boldsymbol{\Phi}}$ can be obtained by calculating the SVD of $\mathbf{Y}$, because they are the eigenvectors of $\mathbf{S}_{\bar{\Phi}}$ that can be obtained from calculating SVD of $\mathbf{Y}$. The SVD of $\mathbf{Y}$ consists of writing it in the form $\mathbf{Y}=\mathbf{U} \boldsymbol{\Sigma} \mathbf{V}^{\mathbf{T}}$, where $\mathrm{U}$ is an $\mathbf{M} \times \mathbf{N}$ of orthonormal columns, $\boldsymbol{\Sigma}$ is an $\mathbf{N} \times \mathbf{N}$ diagonal matrix containing positive elements, and $\mathbf{V}$ an $\mathbf{N} \times \mathbf{N}$ orthonormal matrix. Calculating $\mathbf{Y}^{\mathbf{T}} \mathbf{Y}=\left(\mathbf{U} \boldsymbol{\Sigma} \mathbf{V}^{\mathbf{T}}\right)^{\mathbf{T}} \mathbf{U} \boldsymbol{\Sigma} \mathbf{V}^{\mathbf{T}}=\mathbf{V}(\boldsymbol{\Sigma})^{\mathbf{2}} \mathbf{V}^{\mathbf{T}}$, makes it straightforward that eigenvectors and the corresponding eigenvalues of $\mathbf{S}_{\overline{\mathbf{\Phi}}}=\mathbf{Y}^{\mathbf{T}} \mathbf{Y}$ are equal to the column of $\mathrm{V}$ and the squared elements of the diagonal of $\boldsymbol{\Sigma}$. Hence, the columns of $\mathrm{V}$ are the principal components of $\overline{\boldsymbol{\Phi}}$, and are arranged by descending order based on the corresponding elements of $\boldsymbol{\Sigma}$. The reduced dimensions data matrix $\mathbf{X}$ is obtained by projection of the original data matrix $\boldsymbol{\Phi}$ as follows: $\mathbf{X}=\mathbf{V} \overline{\boldsymbol{\Phi}}$, where $\mathrm{X}$ is a $N \times M$ matrix, same dimensions of the original data matrix $\supsetneqq$. Hence, $L=N \times K$ will be the dimension of the new space in which the data is projected, where $K$ represents the number of selected eigenvalues and vectors to be taken into consideration, based on the amount of information loss, and the required accuracy and tolerance. In our case, $90 \%$ of the information was preserved to represent the original data.

\section{Building the classification model using an SVM-based supervised learning technique}

The support vector machine (SVM) algorithm is a supervised learning technique used in both classification and regression problems, it is a generalization of linear classifiers, as it can deal non linear high dimensional data. Basic SVM-based classifiers are conceived to deal with binary classification. However, for multiple class classification problems such as our case, there exist several extensions, among them, one of the most used is the one versus all approach. Based on the problem formulation, the data needs to be classified into three classes: $\mathrm{s} / \mathrm{p}$, left, and right lateral. In the one versus all approach, one class is taken as class 1, and all the two remaining classes are class 2. Accordingly, the behavior of the data is studied, and fitted to a Gaussian Kernel for classification. SVM classifiers can only deal with classification of two classes, but with the added one versus all approach, it can be adopted to classify between more classes i.e. 3 in our case.

Having the training data, a hyperplane $f(x)$ is established to separate between the two classes, where $x$ is the feature vector, a row of the reduced data matrix $\mathbf{X}$. The function $f(x)$ is a linear discriminant function having the form $\mathbf{f}(\mathbf{x})=\mathbf{w}^{\mathbf{T}} \mathbf{x}+\mathbf{c} \quad \mathbf{w} \in \mathbb{R}^{\mathbf{K}}, \mathbf{c} \in \mathbb{R}$, where $\precsim$ known as weight vector (normal to the hyperplane), and $\mathrm{c}$ is the bias. Let $y_{i} \in\{-1,1\}$ be the label associated to a given sample vector $x_{i}$, where $\mathrm{i}=1, \ldots, \mathrm{N}$. Hence the linear discriminant function $f(x)$ takes values accordingly as shown in equation (2).

$$
f\left(x_{i}\right)= \begin{cases}\geq 0 & y_{i}=+1 \\ <0 & y_{i}=-1\end{cases}
$$

Considering two different points in the L-dimensional feature space, each one belonging to one of the two classes. A margin is the minimal distance separating the decision surface from a sample. The problem consists of finding $f$ that maximize the margin $\mathbf{m}$ and satisfying $\mathbf{y}_{\mathbf{i}}\left(\mathbf{w}^{\mathbf{T}} \mathbf{x}_{\mathbf{i}}+\mathbf{c}\right) \geq \mathbf{m}$. Hence, generalizing for all training patterns, the problem can be declared as follows: find the hyperplane $\mathbf{H}(\mathbf{w}, \mathbf{c}): \mathbf{w}^{\mathbf{T}} \mathbf{x}+\mathbf{c}=\mathbf{0}$, that maximizes the margin $\mathbf{m} /\|\mathbf{w}\|$ and satisfies $\mathbf{y}_{\mathbf{i}}\left(\frac{\mathbf{w}^{\mathbf{T}} \mathbf{x}_{\mathbf{i}}}{\|\mathbf{w}\|}+\frac{\mathbf{c}}{\|\mathbf{w}\|}\right) \geq \frac{\mathbf{m}}{\|\mathbf{w}\|}$. The distance separating each of them from the separating hyperplane is equal to $\frac{1}{\|w\|}$, and the distance between them is $\frac{2}{\|w\|}$ under the assumption of $f(x)$ and the two decision boundaries are equal to \pm 1 (Canonical hyperplanes). For an optimal separability between the two classes, the goal is to maximize the margin between the two classes, i.e. maximizing the distance between any two points belonging to different classes, this can be defined by the equation (3).

$$
\begin{array}{rr}
\underset{\{w, c\}}{\operatorname{Maximize}} & \min \left\|x-x_{i}\right\|: x \in \mathbb{R}^{K}, \\
& w^{T} x+c=0, i=1, \ldots, N .
\end{array}
$$

Solving the optimization problem in equation (3) is equivalent to solving the equation Maximize $\{w, c\} \quad \frac{1}{2}\|w\|^{2}$, subject to $y_{i} \cdot\left(w^{T} x+c\right) \geq 1, \quad i=1, \ldots, N$. where $\|$.$\| is the norm of$ the vector. A usual method used in solving such constrained optimization problem is the Lagrangian multiplier method [13]. It consists of introducing a new variable $\alpha_{i}$ for the sake of converting a given constrained optimization problem into a system of equations, mainly the Lagrangian function (equation (4)), and it's derivative with respect to the primal variables $\{w, b\}$ respectively equal to $0, \frac{\mathbf{d L}}{\mathbf{c}}=\mathbf{0} ; \quad \frac{\mathbf{d L}}{\mathbf{d} \mathbf{w}}=\mathbf{0}$, while

$$
L(w, c, \alpha)=\frac{1}{2}\|w\|^{2}-\sum_{i=1}^{N} \alpha_{i}\left(y_{i} \cdot\left(w^{T} x+c\right)-1\right)
$$

which can be equivalently written as $\mathbf{w}=\sum_{\mathbf{i}=\mathbf{1}}^{\mathbf{N}} \alpha_{\mathbf{i}} \mathbf{y}_{\mathbf{i}} \mathbf{x}_{\mathbf{i}}, \quad \sum_{\mathbf{i}=\mathbf{1}}^{\mathbf{N}} \alpha_{\mathbf{i}} \mathbf{y}_{\mathbf{i}}=\mathbf{0}$. Based on the optimization complementary conditions provided by the KarushKuhnTucker method [14], the constraint must have null coefficients $\alpha_{i}$, as $\alpha_{\mathbf{i}}\left(\mathbf{y}_{\mathbf{i}} \cdot\left(\mathbf{w}^{\mathbf{T}} \mathbf{x}_{\mathbf{i}}+\mathbf{c}\right)-\mathbf{1}\right)=\mathbf{0}, \mathbf{i}=\mathbf{1}, \ldots, \mathbf{N}$. Due to the non linearity of the data, it is more convenient 
to solve the dual problem instead the primal one, and it is the only method to train SVM. Accordingly, replacing $\mathbf{w}=\sum_{\mathbf{i}=\mathbf{1}}^{\mathbf{N}} \alpha_{\mathbf{i}} \mathbf{y}_{\mathbf{i}} \mathbf{x}_{\mathbf{i}}, \quad \sum_{\mathbf{i}=\mathbf{1}}^{\mathbf{N}} \alpha_{\mathbf{i}} \mathbf{y}_{\mathbf{i}}=\mathbf{0}$ into (4) yields to the formulation of the problem, as shown in equation (5), and (6).

$$
\begin{gathered}
\underset{\alpha}{\operatorname{Maximize}} \quad \sum_{i=1}^{N} \alpha_{i}-\frac{1}{2} \sum_{i=1}^{N} \sum_{j=1}^{N} \alpha_{i} \alpha_{j} y_{i} y_{j} w^{T} x_{i} \\
\text { s.t } \quad \alpha_{i} \geq 0, \quad i=1, \ldots, N \quad \sum_{i=1}^{N} \alpha_{i} y_{i}=0
\end{gathered}
$$

Hence, a mapping of the data is done as $\mu: \mathbb{R}^{L} \Longrightarrow F$, where $\mu$ is the mapping function of the input data into a higher dimensional space, and $\mathrm{F}$ is the high dimensional feature space. The training phase depends on the dot product of the mapping $\left\langle\mu\left(x_{i}\right), \mu\left(x_{j}\right)\right\rangle$ in $\mathrm{F}$ instead of $\left\langle x_{i}, x_{j}\right\rangle$. Accordingly, the aforementioned Gaussian kernel $K\left(x_{i}, x_{j}\right)=$ $\left\langle\mu\left(x_{i}\right), \mu\left(x_{j}\right)\right\rangle$ is only known with no need to know what is explicitly the mapping $\mu$. The same steps in equations (5), and (6) are then employed but to the transformed data instead of the original one. The final formulation of the optimization problem would be as shown in equation (7), and (8).

$$
\begin{gathered}
\underset{\alpha}{\operatorname{Maximize}} \quad \sum_{i=1}^{N} \alpha_{i}-\frac{1}{2} \sum_{i=1}^{N} \sum_{j=1}^{N} \alpha_{i} \alpha_{j} y_{i} y_{j} K x_{i}^{T} x_{j} \\
\text { s.t } \quad \alpha_{i} \geq 0, \quad i=1, \ldots, N, \quad \sum_{i=1}^{N} \alpha_{i} y_{i}=0
\end{gathered}
$$

Hence, the discriminant function used for decision is $f(x)=$ $\operatorname{sign}\left(\sum_{i=1}^{N} \alpha_{i} y_{i} K\left(x, x_{i}\right)+b\right)$.

\section{10-Fold Cross-Validation}

Cross validation is a method used in model validation to assess to what extent the results obtained can be generalized, and to avoid the overfitting model in one set of data and loosing generality which degrades the performance when running with new data. Particularly in machine learning, it helps evaluating the predictive performance of the model, and makes the training phase more confident. The training data was divided into 10 subsets and cross validation was applied as shown in the block diagram in Figure 2.

The Cohen's Kappa coefficient indicates how much the

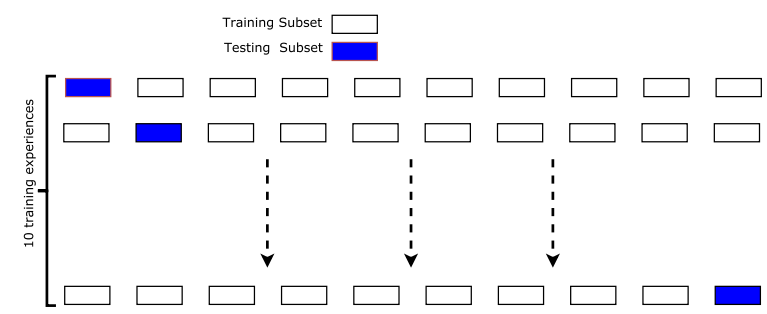

Fig. 2: Scheme of the 10-Fold cross validation applied on the training set.

classifier agrees with truth but while taking into account the agreement by chance, and ignoring it, in order to reflect the intended agreement. The kappa coefficient $\kappa$ is calculated as $\kappa=\frac{P_{a}-P_{e}}{1-P_{e}}[15]$, where $P_{a}$ is the observed agreement,calculated as the ratio of the true positive classifications over the total number of the observations. $P_{e}$ is the agreement by chance or the expected agreement, $\mathbf{P}_{\mathbf{e}}=\frac{1}{\mathrm{n}} \sum_{\mathbf{i}=1}^{3}\left[\frac{\mathrm{ft}_{1} * \mathrm{fc}_{1}}{\mathbf{n}}+\frac{\mathrm{ft}_{2} * \mathrm{fc}_{2}}{\mathbf{n}}+\frac{\mathrm{ft}_{3} * \mathrm{fc}_{3}}{\mathbf{n}}\right]$, where $f t_{i}$ is the marginal frequency of the true class $i$, and $f c_{i}$ is the marginal frequency of the class $i$ as classified by the SVM model

\section{E. Fourth Posture Classification Using Image Processing}

Based on the model built previously, the sleeping posture is classified into one of the three aforementioned classes. If the posture classified is $\mathrm{s} / \mathrm{p}$, so it can be whether supine or prone. Now we are interested to discriminate between prone and supine sleeping postures.

One of the most discriminative properties between prone and supine position is the respiration. It is because we are dealing with the problem as a pattern recognition problem, hence no information about the pressure distribution on the mattress. Respiratory-induced pattern changes between consecutive frames can discriminate between supine and prone positions. That is why, a simple time wise thresholding on the mean of the $\Delta_{i}$ matrix is used to classify prone and supine positions where $\Delta_{i}$ can be expressed by $\Delta_{i}=F_{i}-F_{i-1}$, where $\mathrm{F}$ is the current frame.

\section{F. IoT related requirements for remote medical monitoring}

In the current subsection, we look closer on the communication part of the proposed system. Before expanding design details and the related constrained parameterization, by looking in the Figure 1, it is conveniently noted that the system's framework is based on the core definition of IoT, which consists in a network of connected devices through a secure service layer (SSL), in order to capture and share vital data.

To preserve a reliable communication scheme, the requirements to be considered can be divided in categories [4] as follows:

- Data acquisition: the mattress system's sampling frequency range up to $26 \mathrm{~Hz}$ as a hardware specification. However, medical factors impose a minimum sampling frequency of $2 \mathrm{~Hz}$ which is the minimum value that allows an appropriate detection of respiratory induced pattern changes, which validates the choice of a $2 \mathrm{~Hz}$ as an optimal sampling frequency because one of the essential constraints to comply with in an IoT application requires the system to be an Energy-optimized Solution, specially regarding the amount and quality of data exchange and analysis, which grants a proper Semantic interoperability and data management [16], hence the lowest possible data rate/sampling frequency has to be set.

- Protocol designation : as shown in Figure 1, two communications are involved in the system's operations. The first consists of the communication between the sensor 


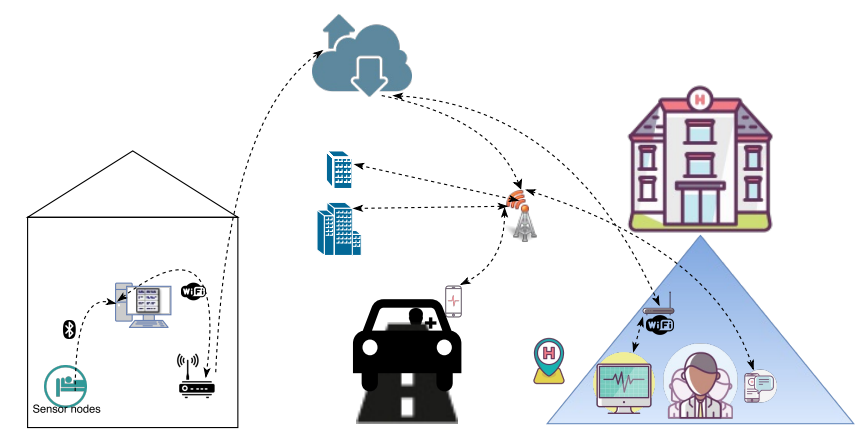

Fig. 3: Internet of Things scenario for remote medical monitoring.

nodes and the workstation, and the second between the workstation and the end-monitoring party. A proper protocol designation for each of the two communications is detailed by assessing the constraints imposed by the application.

Firstly, the heavy (raw) data transfer takes part between the sensor nodes and the workstation, which makes it better to allocate a proper local communication protocol for short distances, and perform the processing locally, before sending small size decision data via internet which is adequate for data consumption. Hence, not only a low data rate communication protocol is sufficient (as shown in the previous point), but also a short distance communication is enough between the sensor nodes and the workstation. Thus, the communication between the sensing mattress and the workstation is chosen to be performed by Bluetooth as an energy efficient potential candidate, with a broadcast frequency of $2.4 \mathrm{GHz}$, which is the short-range radio frequency band, globally unlicensed (but not unregulated) Industrial, Scientific and Medical (ISM) band. Bluetooth is a specification for wireless personal area networks (PANs) as was standardized in the IEEE 802.15.1 standard. Accordingly, the system's communication is assured by a wireless transmitter, having the specifications as shown in the Table I.

Secondly, it is important to ensure mobility for the end-monitoring party, making the communication easier, faster, and almost location-unconstrained. Hence, the second communication part requires wireless Internet connectivity, which can be provided, depending on the location, via several wireless Internet access technologies such as mobile networks technologies, Wireless borad-

\begin{tabular}{|l|c|}
\hline Wireless Standard & Bluetooth \\
\hline Broadcast Frequency & $2.4 \mathrm{GHZ}$ (ISM) \\
\hline Maximum Sensors Acquisition Speed & $200 \mathrm{~Hz}$ \\
\hline Maximum Display Sampling Frequency & $26 \mathrm{~Hz}$ \\
\hline Battery life & 8 Hours (Extendable) \\
\hline
\end{tabular}

TABLE I: Wireless transmission and system's technical specifications. band by Wirelsss Internet Server Providers (WISPs), Satellite communication, or other cable access technologies when connected to a Wi-Fi module for wireless Internet broadcasting.

- Protocol conversion: the Bluetooth network in the application layer (Patient's bed location) is based on a Wireless Sensor Network (WSN) protocol, while the Internet network is TCP/IP based. Hence, an IoT Gateway system is required to receive, analyze, and re-package the acquisition data based on WSN protocols, then capsulate and send the re-packaged data following telecommunication protocols. The workstation is not only where the data processing is performed but it also contains the said IoT gateway to ensure the protocol-diversified communication between both application and perception layers, via the transmission layer.

Accordingly, a brief description of the system is provided as follows. Raw pressure sensing images acquired via built-in pressure sensor nodes in the mattress are sent by Bluetooth to the workstation with a sampling frequency of $2 \mathrm{~Hz}$. In the workstation $(\mathrm{PC})$, the data is processed by the machine algorithm detailed in the previous subsection and decision data is generated. The computer is connected to the internet via Wi-Fi using any Internet access technology, allowing remote monitoring and communicating the decision data. A data storage is provided along with a Web-based cloud, allowing a fast and easy access for the stored data.

For a better illustration, the Internet of Things scenario is shown in Figure 3, showing the connected clinical environment (e.g, patient's home, hospital, and doctor.), allowing a proper communication to serve and improve medical services.

\section{Simulation RESUlts}

The presented algorithm was tested on completely new data containing 90 samples that have never been seen before. The sampling frequency used is $2 \mathrm{~Hz}$, to make sure that respiratory induced pattern changes are covered. The concept of pattern recognition with such low computation cost using the SVM algorithm made the system work in a quasi real time basis. As shown in the training model confusion matrix in Table 
TABLE II: Training model confusion matrix

\begin{tabular}{cccc} 
Class & S/P & L.L & R.L \\
\hline S/P & 70 & 3 & 1 \\
L.L & 1 & 69 & 4 \\
R.L & 1 & 3 & 70 \\
\hline Recall/Sensitivity & 94.5 & 93.2 & 94.5 \\
Precision & 97.22 & 93.2 & 94.59 \\
Specificity & 98.64 & 95.94 & 97.27 \\
Accuracy & & 94.14 & \\
\hline
\end{tabular}

1, a high predictive performance was obtained. As seen, the model classify $100 \%$ the s/p class, whereas for left and right lateral, a slight confusion exists.

Furthermore, to measure the performance of the classifier on completely new data, a 90 new samples that have never been used are also fed in the classifier and the performance is shown in the confusion plot in Figure 4.

The obtained $\kappa$ is 0.866 showing and validating the obtained results and the high predictive performance of the model. Moreover, a counter indicating the number of tosses

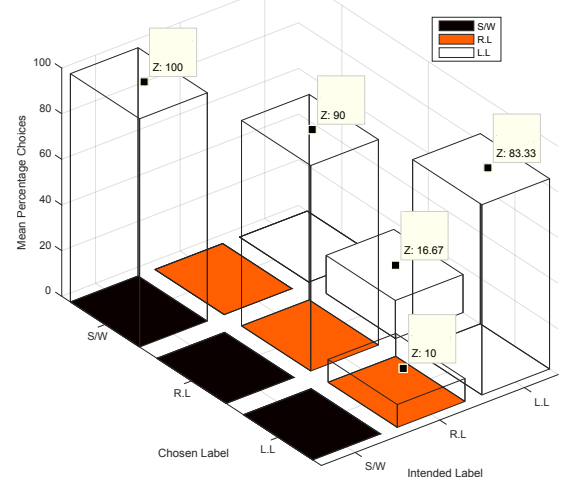

Fig. 4: SVM predictive performance for new data: confusion plot.

occurring during the time of recording has been implemented as an extension to the algorithm. Such counter could be used in various applications where the number of posture change is required during a recording, for example for assessing the sleep quality.

Finally, the emerging 5G technology involving diverseness in devices communication, along with an Heterogeneous Networking (HetNet) paradigm, makes the communication between several types of wireless access nodes more accessible, even those having distinct backhaul connectivity, design parameters, and coverage [17]. Which opens perspectives for improvements of such scenarios, to ensure fast, reliable, secure, and standardized communication and operations involving remote monitoring in the medical field.
IV. CONCLUSION

A novel approach for posture monitoring using body pressure distribution on the mattress is proposed in this paper. The obtained results show a high accuracy of detection with a Cohen's Kappa coefficient $\kappa=0.866$. The objective of the present work is to reduce as much as possible the computation cost, and the requirement for storage in the aim of making it suitable for a wider range of applications, specially the ones involving long term monitoring. Future work will focus on two main goals: hardware implementation and more insights on the design optimization, and further investigation of the physical monitoring problem applied in the area of sleep studies, especially trying to explore how such automated surveillance could be a useful tool for sleep quality assessment.

\section{REFERENCES}

[1] G. H. Enderby, "Controlled circulation with hypotensive drugs and posture to reduce bleeding in surgery preliminary results with pentamethonium iodide," The Lancet, vol. 255, no. 6617, pp. 1145-1147, 1950.

[2] M. Abouzari, A. Rashidi, J. Rezaii, K. Esfandiari, M. Asadollahi, H. Aleali, and M. Abdollahzadeh, "The role of postoperative patient posture in the recurrence of traumatic chronic subdural hematoma after burr-hole surgery," Neurosurgery, vol. 61, no. 4, pp. 794-797, 2007.

[3] H. Kopetz, "Internet of things," in Real-time systems. Springer, 2011, pp. 307-323.

[4] Q. Zhu, R. Wang, Q. Chen, Y. Liu, and W. Qin, "Iot gateway: Bridgingwireless sensor networks into internet of things," in Embedded and Ubiquitous Computing (EUC), 2010 IEEE/IFIP 8th International Conference on. IEEE, 2010, pp. 347-352.

[5] D. Niyato, E. Hossain, and S. Camorlinga, "Remote patient monitoring service using heterogeneous wireless access networks: architecture and optimization," IEEE Journal on Selected Areas in Comun, vol. 27, no. 4, pp. 412-423, 2009.

[6] L. Atzori, A. Iera, and G. Morabito, "The internet of things: A survey," Computer networks, vol. 54, no. 15, pp. 2787-2805, 2010.

[7] S. R. Islam, D. Kwak, M. H. Kabir, M. Hossain, and K.-S. Kwak, "The internet of things for health care: a comprehensive survey," IEEE Access, vol. 3, pp. 678-708, 2015.

[8] K. Nicol and D. Rusteberg, "Pressure distribution on mattresses," Journal of biomechanics, vol. 26, no. 12, pp. 1479-1486, 1993.

[9] J. W. DeVocht, D. G. Wilder, E. R. Bandstra, and K. F. Spratt, "Biomechanical evaluation of four different mattresses," Applied Ergonomics, vol. 37, no. 3, pp. 297-304, 2006.

[10] R. Yousefi, S. Ostadabbas, M. Faezipour, M. Nourani, V. Ng, L. Tamil, A. Bowling, D. Behan, and M. Pompeo, "A smart bed platform for monitoring \& ulcer prevention," in 4th International Conference on Biomedical Engineering and Informatics (BMEI), vol. 3. IEEE, 2011, pp. 1362-1366.

[11] H.-C. Hsu and R.-C. Lo, "A new mattress development based on pressure sensors for body-contouring uniform support," arXiv preprint arXiv:1308.2196, 2013.

[12] S. Products, "Real time tactile temperature analysis temperature sensor," http://www.sensorprod.com/pdf/mattress-tem_brochure.pdf, 2006, accessed: 16-May-2016.

[13] R. T. Rockafellar, "Augmented lagrange multiplier functions and duality in nonconvex programming," SIAM Journal on Control, vol. 12, no. 2, pp. $268-285,1974$.

[14] J. Kyparisis, "On uniqueness of kuhn-tucker multipliers in nonlinear programming," Mathematical Programming, vol. 32, no. 2, pp. $242-$ $246,1985$.

[15] M. L. McHugh, "Interrater reliability: the kappa statistic," Biochemia medica, vol. 22, no. 3, pp. 276-282, 2012.

[16] D. Miorandi, S. Sicari, F. De Pellegrini, and I. Chlamtac, "Internet of things: Vision, applications and research challenges," Ad Hoc Networks, vol. 10, no. 7, pp. 1497-1516, 2012.

[17] M. R. Palattella, M. Dohler, A. Grieco, G. Rizzo, J. Torsner, T. Engel, and L. Ladid, "Internet of things in the 5g era: Enablers, architecture, and business models," IEEE Journal on Selected Areas in Communications, vol. 34 , no. 3 , pp. 510-527, 2016. 